\title{
Maximizing the Impact of Air Bubbles for Membrane Fouling Control in Microalgae Harvesting
}

(Date Received: 25.4.2017/Date Accepted: 29.07.2017)

Arthur Eliseus, Dr Muhammad Roil Bilad"

Chemical Engineering Department, Universiti Teknologi PETRONAS, Bandar Seri Iskandar, 32610 Perak, Malaysia.

*Corresponding Author: mroil.bilad @utp.edu.my;Tel.: +6053687646.

\section{ABSTRACT}

Harvesting microalgae using membrane is challenging due to the nature of microalgae having very high membrane fouling potential. Numerous techniques have been proposed for membrane fouling control, including optimizing operational cycles, imposing shear-rates via air bubbles and dosing chemicals for feed conditioning and membrane cleaning. As an established method, the efficacy of air bubbles for membrane fouling control can be improved by maximizing the impact of shear-rates in scouring foulant from the membrane surface. In this study, we investigate the effect of tilting angles, switching periods as well as aeration rates in a lab-scale submerged filtration system by filtering microalgae solution. Results showed that higher tilting angles improve the cleaning efficiency by offering higher flux of up to 2.7 times at an angle of 20 as opposed to the vertical one. It was also found that operating a one-sided panel (without switching) was about $20 \%$ better than a two-sided panel, in which the latter involved switching mode to offer aeration of both panel sides. This technique is effective in controlling fouling and can lead to energy saving for full-scale modules.

Keywords: Air Bubbles, Membrane Fouling, Membrane Photobioreactor, Shear-Rate, Tilted Module

\subsection{INTRODUCTION}

Membrane-based technology have been proven effective to harvest microalgae as it ensures thorough biomass retention and offers a cost-effective option compared to other energyconsuming methods [1]. However, membrane fouling remains the major challenge in microalgae harvesting using membrane. Its effectiveness is dictated by the ability to manage fouling. Membrane fouling diminishes permeability in a long run, if not managed properly, to an extent that a filtration system is uneconomically feasibly. Various techniques have been introduced to manage fouling. Among them are by lowering of operational flux, implementing periodical cleanings or imposing shear-rates via air bubbles aeration [2]. All of them partly effective, but further improvements are still possible to offer a better membrane fouling control.

Advantage of air bubbles in controlling membrane fouling has been acknowledged, and it has become a standard method for membrane fouling control in various membrane processes, particularly in submerged membrane bioreactor (MBR) for wastewater treatment. The bubbles induce hydrodynamic force that flows atop the membrane surface and promotes tangential shear-rates [3]. The local shear transients mitigate fouling formation by constant removal of foulant from the membrane surface [4]. Generally, the hydrodynamic powers induced by the air bubbles are drag-force and lift-force, which are closely dealing with filtrate flow, cross-flow velocity and near-wall velocity gradient.

Membrane fouling mitigation via air bubbles is largely affected by the size of the bubbles, particularly effective under slug flow condition at very high cross-flow velocities [5]. The effectiveness of slug-flow, however, must be compensated by a high energy required to pump required high volumetric flow rates of the air bubbles. Sparging with large pulse bubbles improves fouling control compared to coarse bubbles [6], [7]. Continuous air bubbling is more effective than intermittent bubbling [8]. For the former, air bubbles continuously scour-off the foulant, while the latter fouling occurs easily during aeration relaxation. Moreover, higher air bubbling intensity is required in the intermittent bubbling to compensate the relaxation time if similar hydraulic productivity is targeted. Despite of all aforementioned advantages, air bubble scouring has few inherent limitations, as summarized elsewhere [9]. They only produce relatively weak shear rates, the impact reaches a "plateau" at a certain air supply, and most importantly it is difficult to ensure directions and distributions of bubbles flow paths on the membrane surface. Air bubbles tend to reside in the center-space between adjacent panels (the furthest distance from the membrane surface).

As an attempt to improve air bubbles efficacy, an inventive bubble generator device was proposed [3]. They claim that an ejector-type bubble generator (called bubble generator plate) is highly effective for fouling control during harvesting of Chlorella sp. KR-1 by doubling the membrane permeability. One of the main reasons for the effectiveness, in our opinion, is because of the cell design that allows maximum contact of generated air bubbles with the membrane surface. It is achieved by placing the membrane horizontally (active layer facing down) and the air bubbles is supplied from underneath, allowing maximum contact of air. The effect is also enhanced by cross-flow velocity. Nevertheless, despite of its effectiveness, we believe that this arrangement is impractical in a full-scale module.

In the present study, a tilted membrane module is proposed to maximize the contacts of air bubbles with membrane surface and thus improve their efficacy for membrane fouling control. As the tilting angle increases, higher impact between the air bubbles and membrane surfaces is expected, hence scouring off the deposited foulant. The tests were conducted in a lab-scale submerged filtration treating microalgae solution cultivated in 


\section{MAXIMIZING THE IMPACT OF AIR BUBBLES FOR MEMBRANE FOULING CONTROL IN MICROALGAE HARVESTING}

secondary effluent medium. The effect of fouling was studied and the filtration analysis was conducted by obtaining the permeability of membrane by varying tilting angles of the panel, assessing the switching periods, and investigating the effect of aeration rates. Lastly, we assess the feasibility of the new concept and compared with few established full-scale submerged membrane modules.

\subsection{MATERIALS AND METHODS}

\subsection{Membrane preparation and characterization}

Phase inverted PVDF membrane was prepared via immersion precipitation method using PVDF (Arkema, Mw=300 kDa), dimethylacetamide (DMAC, Sigma-Aldrich) and demineralized water as the polymer, solvent \& non solvent, respectively. After being thoroughly dissolved and degassed, the polymer solution (mixture of $15 \%$ wt of the polymer in the solvent) was cast using a doctor blade with net casting thickness of $220 \mu \mathrm{m}$ at room temperature \& humidity atop a non-woven support (Noratexx 2471). The cast film was then immediately immersed in a bath containing demineralized water in which phase separation occurs. The membrane was stored wet until usage. The PVDF membrane was be assembled into a working module with an effective membrane area of $0.012 \mathrm{~m} 2$ (1 sided surface of $10 \mathrm{~cm}$ $\mathrm{x} 12 \mathrm{~cm}$ frame) before used.

The microstructure images of the membrane were obtained using scanning electron microscope. The images were further processed by ImageJ (NIH) to estimate surface porosity, pore size and pore density. The pore size and distribution were also measured using capillary flow porosimetry. Goniometer, micrometer and dry wet method were used for measuring contact angle, fibers thickness and porosity, respectively. The thickness $(\mathrm{mm})$, maximum pore size $(\mu \mathrm{m})$, mean pore $(\mu \mathrm{m})$, contact angle $\left({ }^{\circ}\right)$, clean water permeability $\left(\mathrm{L} / \mathrm{m}^{2} \mathrm{hbar}\right)$, surface pore size $(\mu \mathrm{m})$, surface pore density (pores $/ \mu \mathrm{m}^{2}$ ), and lastly surface porosity $(\%)$ of the membranes are $0.18,0.19,0.16,75 \pm 5,494,0.1,19$ and 11 , respectively.

\subsection{Experimental Set-up}

The submerged filtrations were performed by immersing a filtration panel into a two-liter tank containing microalgae solution. The module was connected to a water manometer and a vacuum pump. The pump was operated at a constant pressure of 0.12 bar to ensure a constant flux operation so that membrane fouling can be monitored from the trend of decreasing of membrane permeability over time. Aeration system was provided at a range of 1.08 to $1.80 \mathrm{~L} / \mathrm{min}$. The air bubble is used to scour off the foulant deposited on the membrane surface hence act as the fouling control.

\subsection{Filtration test}

The clean water permeability $(\mathrm{L})$ of the membrane was measured after it was assembled into a filtration panel in a submerged filtration system. The flux (J) and permeability (L) of the membranes were calculated using eqs. 1 and 2, respectively.

$$
\begin{array}{ll}
\mathrm{J}=\mathrm{V} /(\mathrm{A} \mathrm{t}) & \left(\mathrm{L} / \mathrm{m}^{2} \mathrm{~h}\right) \text { or } \mathrm{LMH} \\
\mathrm{L}=\mathrm{J} / \Delta \mathrm{P} & \left(\mathrm{L} / \mathrm{m}^{2} \mathrm{hbar}\right)
\end{array}
$$

where $\mathrm{V}$ is volume of permeate $(\mathrm{L})$, A effective filtration area $\left(\mathrm{m}^{2}\right)$, $\mathrm{t}$ filtration time $(\mathrm{h})$ and $\Delta \mathrm{P}$ trans-membrane pressure.
When the filtration involves relaxation, the net-permeability and net-flux were calculated by accounting the relaxation time.

The effect of tiling angle was first tested in order to determine the most optimum value. The volume of filtered water was collected every 10 minutes continuously for 2 hours. The evolution of membrane flux as function of time was then monitored. Later, the effect of switching period was also investigated. The switching periods were run at 0 (no switch), 10, 20, and 30 mins. The effect of aeration rate was also studied and the rates were set at 1.08, 1.26, 1.44, 1.62, and $1.80 \mathrm{~L} / \mathrm{min}$. After each test, a chemical cleaning was performed by soaking the membrane into $1 \%$ of sodium hypochlorite $\left(\mathrm{Cloroc}^{\circledR}\right)$ solution for 2 hours in demineralized water.

\subsection{RESULTS AND DISCUSSION}

\subsection{Tilting a plate-and-Frame Module: Practical Implication}

The conventional vertical module panel suffers from low contact of air bubbles with membrane surface (Figure 1). In this alignment, air bubbles tend to move in the center space between two adjacent panels, hence diminishing their scouring roles. When a panel is tilted at a certain angle, it help to maximize contacts of air bubbles with membrane surface, thus maximize bubbles scouring impacts. The tilted panel blocks the upward trajectory of air bubbles due to Buoyancy, and forces the bubbles to travel upward always in-contact with membrane surface where they scour off the foulant via drag force.

Tilting membrane panel requires a change of operational mode by involving switch. When a panel is mounted with two sided membrane, only one side of membrane surface is be aerated, whereas the opposite surface is not exposed by aeration. To ensure both sides are equally aerated, the module shall be operated by involving switch: in a left-mode for a specific period before it is switched to the right-mode (Figure 1). For instance, on the left mode, only A and D sides are aerated, and for the right-mode only $\mathrm{B}$ and $\mathrm{C}$ side are aerated.

\subsection{Effect of Tilting Angle}

Tilting membrane panel significantly improves flux (Figure 2). As the angle increased up to $20^{\circ}$, the flux also increases from 10 (vertical) to $27 \mathrm{LMH}\left(20^{\circ}\right)$. Tilting angle of $20^{\circ}$ (the highest possible applied with our set-up) shows the highest flux of 27.0 $\mathrm{LMH}$ followed by $15^{\circ}, 10^{\circ}, 5^{\circ}$ and $0^{\circ}$ of $25.5,22.0,12.5$ and 10.0 $\mathrm{LMH}$, respectively. This order clearly demonstrates favorable

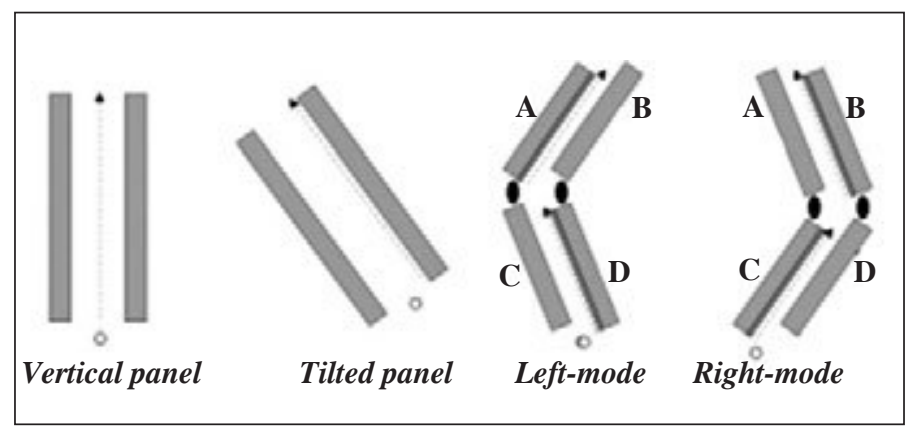

Figure 1: Rationale and implication of panel tilting. The left- and right-modes show the sides of membrane exposed to aeration when operating in switch mode 
effect of shear rate on the membrane surface. As the angle increases, more intense contacts occur between air bubbles and the membrane surface which help to scour off the foulant that had been deposited on the membrane surface.

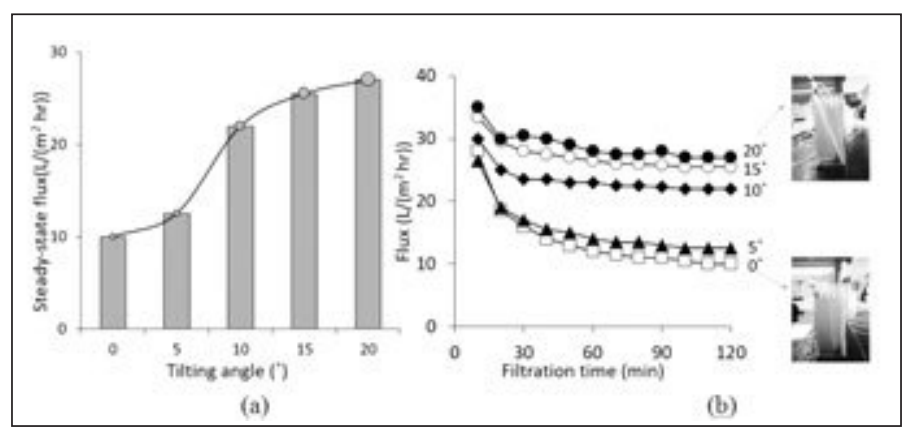

Figure 2: Effect of tilting angle on membrane flux. The tests were conducted at constant pressure of 0.12 bar and aeration rate of 1.8 $L / m i n$. The inset images show the tilting arrangement of the panel in a filtration tank

Tilted panel is proven effective in controlling membrane fouling. It works by removing the foulant deposited on the membrane surface and reducing the rate of foulant to be deposited on the pre-fouled membrane. Based on the results, increasing higher tilting angle will most likely increase the flux further. Figure 2 shows that the flux almost reaches plateau at $20^{\circ}$. Increasing the angle further will require a larger foot-print in order to accommodate more membrane module to retain high packing density. Therefore, the aim is to obtain the smallest yet effective angle. It can be seen in Figure 2 that $15^{\circ}$ and $20^{\circ}$ has only $5 \%$ difference in flux, therefore $15^{\circ}$ was selected as the optimum angle. Despite of the small margin, operating at $15^{\circ}$ still able to offer 2.7 times higher flux to compared to the vertical plate.

\subsection{Effect of Switching Period}

In order to ensure both membrane sides are equally aerated, we introduced a switch mode and investigated its effect. Due to the set up limitation, the experiment was conducted using one-sided membrane and incorporating intermittent aeration. When the switch was on, it indicated that the membrane was exposed to aeration, vice versa. The results show that operating one-sided membrane with continuous aeration without any switch gave the highest flux (Figure 3 left). Moreover, operating at 10, 20 and 30 minute period gave about similar overall flux, about a half of the one without switch.

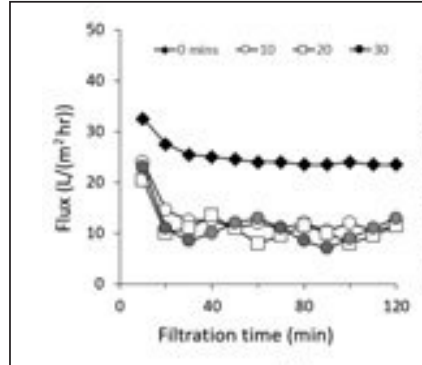

(a)

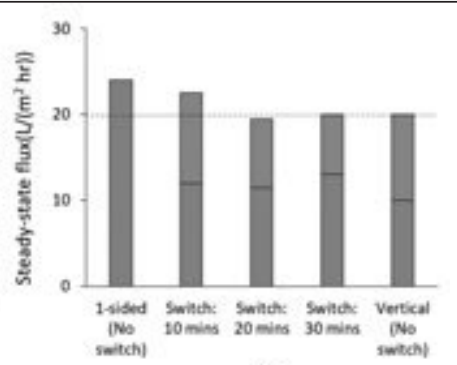

(b)
Figure 3: Effect of aeration switch on membrane flux (a). The red and green colors show the evolution of flux during off and on period, respectively. Panel flux of one side membrane (without switch) performed better than two-side membrane with switch (b)
The operational modes were chosen in order to detect the small changes on fouling rates with respect to their switching period. The maximum period of 30 mins was selected because longer duration may clean one surface effectively but let the opposite side suffers fouling severely. Experiment was conducted with the increment of 10 minutes differences because too-low period is taught to be impractical and lead to high energy demands.

There is a significant flux variation over testing duration at high periods ( $<10$ mins) (Figure 3a). The average flux for each membrane when it is on and off was added in order to obtain a two-sided membrane result. Figure $\mathbf{3 b}$ shows the steady state flux of membrane panel showing that operating one-sided membrane without switch achieve double the overall flux compared to a two sided-membrane with switch, enabling it to achieve the same productivity with a panel with two-sided membrane. It worth to distinct between overall flux and panel flux. The former refer to the one in Eq. 1 and the latter is the sum of flux of both panel sides when aerated and not aerated. The result also confirms that membrane can easily be fouled when it is operated without aeration. Therefore, instead of using twosided membrane panel, we recommend to use a tilted one-sided membrane panel. This can also reduce the energy required for switching and halves the cost of the membranes.

\subsection{Effect of Aeration Rate}

The performance of tilted panel can be further improved by varying the aeration rate (Figure 4). Most of the commercial modules focus on reducing the aeration rate whilst retaining the flux. The flux is generally proportional to the aeration rate until a threshold value at which any increment in aeration rate won't improve the membrane flux. Results show that the permeability of membrane during one hour of filtration at five different aeration rates. One hour is the minimum filtration duration required in order for the membrane to achieve steady state flux. As expected, the final flux was found higher at higher aeration rate and the threshold value was not reached yet. At $1.8 \mathrm{~L} / \mathrm{min}$ aeration rate and at $15^{\circ}$ tilting angle, it gave about 1.8 times higher permeability compared to the vertical plate.

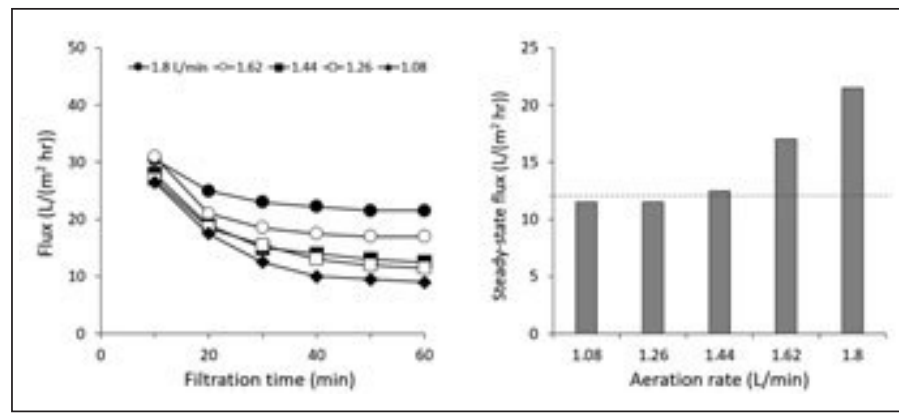

Figure 4: Effect aeration rates on the flux profile (A) steady-state flux after one hour of filtration (B). The flux increases as an increase in aeration rates. The dashed line depicts a flux of vertical panel at an aeration rate of $1.8 \mathrm{~L} / \mathrm{min}$

Application of this system can be compared with the current commercial membrane technologies. Memcor, Zenon and Kubota membranes applied average specific aeration demand with respect to membrane $\left(\mathrm{SAD}_{\mathrm{m}}\right)$ of $0.2,0.33$ and $0.98 \mathrm{Nm}^{3} /$ $\mathrm{m}^{2} \mathrm{~h}$, respectively. In this study, the $\mathrm{SAD}_{\mathrm{m}}$ was found to be 0.45 $\mathrm{Nm}^{3} / \mathrm{m}^{2} \mathrm{~h}$, which lies between the ranges of the commercial modules and proves that the high flux achieved in this study was 


\section{MAXIMIZING THE IMPACT OF AIR BUBBLES FOR MEMBRANE FOULING CONTROL IN MICROALGAE HARVESTING}

not because of over-aeration. This tilted module is proven to be effective and performs better compared to Zenon and Kubota by having lower specific aeration demand with respect to permeate $\left(\mathrm{SAD}_{\mathrm{p}}\right)$. The $\mathrm{SAD}_{\mathrm{p}}$ for Zenon and Kubota was found to be at 27 and $79 \mathrm{~m}^{3}$ air $/ \mathrm{m}^{3}$ permeate, whereas the $\mathrm{SAD}_{\mathrm{p}}$ for this module was as low as $22.5 \mathrm{~m}^{3}$ air $/ \mathrm{m}^{3}$ permeate. This indicates that this module require less aeration in order obtain the same permeation compared to the two commercial products. It is proven as the permeability for the proposed module is $22 \mathrm{LMH}$ which is higher compared to Kubota and similar to Memcor's membranes.

\subsection{CONCLUSIONS}

Tilting the membrane module significantly improves flux because it enhances contacts between air bubbles and the membrane surface and thus air bubbles can scour off the foulant effectively. The flux can be increased to as high as 2.7 times. A tilting angle of $15^{\circ}$ was chosen as the optimum angle in this study because it offers flux improvement as well as reduce module foot-print. Next, switching mode for a two-sided membrane was ineffective because the membrane can easily be fouled when it is not aerated. Hence, operating at one-sided membrane is recommended. The membrane panel in this study was not over-aerated as the aeration rate provided was within the range of aeration given in commercial modules for MBR application. In fact, it was proven that the $\mathrm{SAD}_{\mathrm{p}}$ of the proposed module was found to be lower (22.5 vs $27-29 \mathrm{~m}^{3}$ air $/ \mathrm{m}^{3}$ permeate) compared to the some commercial modules. Despite of having slightly lower in packing density, the proposed module requires less aeration rate which makes the system to be energy saving in terms of overall energy demand.

\section{ACKNOWLEDGMENTS}

We acknowledge Universiti Teknologi PETRONAS for providing grants (grant number: 0153AA-F58 and 0153AAF59) and facilities for conducting the research activities.

\section{REFERENCES}

[1] P. Mouchet and V. Bonnélye, "Solving algae problems: French expertise and world-wide applications," J. Water Supply Res. Technol. - Aqua, vol. 47, no. 3, pp. 125-141, May 1998.

[2] P. Schoeberl, M. Brik, M. Bertoni, R. Braun, and W. Fuchs, "Optimization of operational parameters for a submerged membrane bioreactor treating dyehouse wastewater," Sep. Purif. Technol., vol. 44, no. 1, pp. 61-68, Jul. 2005.

[3] T. Hwang, Y.-K. Oh, B. Kim, and J.-I. Han, "Dramatic improvement of membrane performance for microalgae harvesting with a simple bubble-generator plate," Bioresour. Technol., vol. 186, pp. 343-347, Jun. 2015.

[4] P. Le-Clech, V. Chen, and T. A. G. Fane, "Fouling in membrane bioreactors used in wastewater treatment," J. Membr. Sci., vol. 284, no. 1-2, pp. 17-53, Nov. 2006.

[5] Y. Wu, J. Wang, H. Zhang, H. H. Ngo, W. Guo, and N. Zhang, "The impact of gas slug flow on microfiltration performance in an airlift external loop tubular membrane reactor," RSC Adv., vol. 6, no. 110, pp. 109067-109075, Nov. 2016.

[6] S. Jankhah and P. R. Bérubé, "Pulse bubble sparging for fouling control,” Sep. Purif. Technol., vol. 134, pp. 58-65, Sep. 2014.

[7] S. Chang and A. G. Fane, "The effect of fibre diameter on filtration and flux distribution - relevance to submerged hollow fibre modules," J. Membr. Sci., vol. 184, no. 2, pp. 221-231, Mar. 2001.

[8] J. Tian, Y. Xu, Z. Chen, J. Nan, and G. Li, “Air bubbling for alleviating membrane fouling of immersed hollow-fiber membrane for ultrafiltration of river water," Desalination, vol. 260, no. 1-3, pp. 225-230, Sep. 2010.

[9] M. R. Bilad, D. Vandamme, I. Foubert, K. Muylaert, and I. F. J. Vankelecom, "Harvesting microalgal biomass using submerged microfiltration membranes," Bioresour. Technol., vol. 111, pp. 343-352, May 2012.

\section{PROFILES}

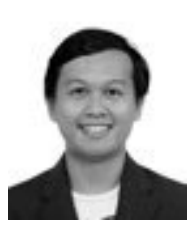

ARTHUR ELISEUS is currently a degree holder of Bachelor of Chemical Engineering in Universiti Teknologi PETRONAS (2012-2017). His main role in this project concentrates on experimental design and data analysis for different parameters in membrane fouling control. Email: arthureliseus@gmail.com.

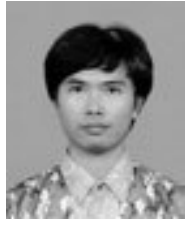

DR MUHAMMAD ROIL BILAD has 12+ years of research experience on membrane science and technology. He has published over 50 ISI highimpact journal publications, inventor/co-inventor of 3 patents and has delivered $25+$ technical talks. Before joined as faculty member in Universiti Teknologi PETRONAS (UTP), he worked as scientist at the Centre for Surface Chemistry and Catalysis at KU Leuven Belgium (2012-2013), from where he completed his doctoral degree in bioscience engineering. During his tenure at Masdar Institute of Science and Technology UAE (20132015), he developed distillation membrane and advanced membrane modules. Later, he also worked at Singapore Membrane Technology Centre NTU Singapore (2015-2016), where he developed novel forward osmosis module. Since May 2016 after joining UTP, he is establishing his own research on membrane science and technology in Chemical Engineering Department. Email: mroil.bilad@utp.edu.my. 